\title{
Highly sensitive on-site detection of glucose in human urine with naked eye based on enzymatic-like reaction mediated...
}

Article in Biosensors \& Bioelectronics · September 2016

DOI: 10.1016/j.bios.2016.09.090

CITATION

1

5 authors, including:

Zhiyang Zhang

Humboldt-Universität zu Berlin

18 PUBLICATIONS 233 CITATIONS

SEE PROFILE

\section{Lingxin Chen}

Chinese Academy of Sciences

271 PUBLICATIONS 7,542 CITATIONS

SEE PROFILE
READS

67

\section{Zhaopeng Chen}

Chinese Academy of Sciences

34 PUBLICATIONS 997 CITATIONS

SEE PROFILE

Some of the authors of this publication are also working on these related projects:

The Natural Science Research Foundation of China, Grant No. 21575159, "The analysis method for the intracellular active species interactive changes under typical heavy metal ions stress by biological optical imaging technology". View project

The Natural Science Research Foundation of China, Grant No. 21275158, "Study on the construction of functionalized nano optical probes for probing forms of trace heavy metal pollutants in water". View project 


\title{
Highly sensitive on-site detection of glucose in human urine with naked eye based on enzymatic-like reaction mediated etching of gold nanorods
}

\author{
$\underline{\text { Zhiyang Zhang }}^{\mathrm{a}, \mathrm{b}}$, Zhaopeng Chen ${ }^{\mathrm{a}, *}$, Fangbin Cheng ${ }^{\mathrm{c}}$, Yaowen Zhang ${ }^{\mathrm{a}, \mathrm{b}}$, Lingxin Chen ${ }^{\mathrm{a}}$ \\ ${ }^{a}$ Key Laboratory of Coastal Environmental Processes and Ecological Remediation, Yantai Institute of Coastal Zone Research (YIC), Chinese Academy of \\ Sciences (CAS); Shandong Provincial Key Laboratory of Coastal Environmental Processes, YICCAS, Yantai, Shandong 264003, PR China \\ b University of Chinese Academy of Sciences, Beijing 100049, PR China \\ c Ocean school, Yantai University, Yantai 264005, PR China
}

\section{A R T I C L E I N F O}

\section{Keywords:}

Colorimetric detection

Urine glucose

Gold Nanorods

Etching

Molybdate

\begin{abstract}
A B S T R A C T
Based on enzymatic-like reaction mediated etching of gold nanorods (GNRs), an ultrasensitive visual method was developed for on-site detection of urine glucose. With the catalysis of $\mathrm{MoO}_{4}{ }^{2-}$, GNRs were efficiently etched by $\mathrm{H}_{2} \mathrm{O}_{2}$ which was generated by glucose-glucose oxidase enzymatic reaction. The etching of GNRs lead to a blue-shift of logitudinal localized surface plasmon resonance of GNRs, accompanied by an obvious color change from blue to red. The peak-shift and the color change can be used for detection of glucose by the spectrophotometer and the naked eyes. Under optimal condition, an excellent sensitivity toward glucose is obtained with a detection limit of $0.1 \mu \mathrm{M}$ and a visual detection limit of $3 \mu \mathrm{M}$ in buffer solution. Benefiting from the high sensitivity, the successful colorimetric detection of glucose in original urine samples was achieved, which indicates the practical applicability to the on-site determination of urine glucose.
\end{abstract}

\section{Introduction}

Statistics indicate that about 382 million people were suffering from diabetes in 2013 and the number to rise to 592 million by 2035 (Guariguata et al., 2014). The blood glucose is usually used as a clinical indicator of diabetes. However, it is inconvenient and painful to draw blood by hand pricking and this procedure may lead to infection. In contrast, the use of urine glucose is simpler and safer. In addition, the presence of glucose in urine is more direct indicator for a more dangerous condition of diabetes. When the concentration of glucose in urine is more than $500-1000 \mathrm{mg} / \mathrm{L}(2.8-5.6 \mathrm{mM})$, the urine test is positive (Urakami et al., 2008). In consideration of its convenience, painlessness and affordability, urine glucose monitoring may be a good alternative, especially in low-income regions. The development of colorimetric assay for urine glucose detection has attracted much attention because of its low cost, visuality and potential for on-site/ in-home application. Even though many colorimetric glucose sensors have achieved satisfactory sensitivity, yet the real practicability is still limited by the need of complex sample pretreatment, such as centrifugal separation (Bo et al., 2013; Su et al., 2012, 2015a; Zheng et al., 2015).

Gold nanoparticles have been widely applied to various colorimetric methods because they exhibit strong localized surface plasmon reso- nance (LSPR) within the visible range (Saha et al., 2012). Typically, such colorimetric sensors are based on red-shift of LSPR which is induced by nanoparticles aggregation (Saha et al., 2012). Gold nanorods (GNRs) have gained increasing attention in fabrication of nonaggregation-based colorimetric sensors (Liu et al., 2013; Saa et al., 2014; Zhang et al., 2015a, 2014a, 2014b, 2015b) based on shapedependent LSPR property (Pérez-Juste et al., 2005). In contrast to aggregation-based sensors, this kind of sensors possesses obvious advantages: (1) they are label-free, avoiding the modification of nanoparticles; (2) they don't face the false positive signal from autoaggregation; (3) they can be made into test paper; (Zhang et al., 2014b) and (4) GNRs have tunable plasmonic bands and high stability. Most recently, some researchers have successfully applied GNRs for glucose sensing (Liu et al., 2013; Saa et al., 2014). Specifically, Liu et al., first proposed a colorimetric method for glucose detection in human serum based on Fenton reaction-mediated etching of GNRs. However, this method faces high detection limit $(1000 \mu \mathrm{M})$, long incubation time (more than $1 \mathrm{~h}$ ) and the centrifugation of serum sample. High improvement has been achieved to increase the sensitivity by Saa et al.. They developed a bienzyme system-mediated etching of GNRs method and obtained a lower detection limit of $10 \mu \mathrm{M}$. The drawback is that, the use of additional enzyme will not only increase the cost in storage and transportation, but also the risk of

\footnotetext{
* Corresponding author

E-mail address: zhpchen@yic.ac.cn (Z. Chen).
} 
denaturisation of the enzyme. Furthermore, the two methods still have not achieved on-site detection in the original blood sample (whole blood).

Our recent work indicates that molybdate $\left(\mathrm{MoO}_{4}{ }^{2-}\right)$ can act an efficient catalyst to accelerate the etching of GNRs in the presence of $\mathrm{I}^{-}$ and $\mathrm{H}_{2} \mathrm{O}_{2}$ (Zhang et al., 2015b, 2015a). In this work, we develop an ultrasensitive GNRs sensor for glucose based on the combination of oxidase-catalyzed glucose oxidation and molybdate-catalyzed etching of GNRs by $\mathrm{H}_{2} \mathrm{O}_{2}$. This method shows excellent sensitivity with a detection limit of $0.1 \mu \mathrm{M}$ and is simple to carry out. The aim of this study is to use this method for an on-site detection of urine glucose with naked eye.

\section{Materials and methods}

\subsection{Chemicals}

Hydrogen tetrachloroaurate(III) dehydrate, cetyltrimethylammonium bromide (CTAB), ascorbic acid, glucose, $\mathrm{Na}_{2} \mathrm{MoO}_{4}, \mathrm{H}_{2} \mathrm{O}_{2}$, $\mathrm{NaBH}_{4}$ and $\mathrm{AgNO}_{3}$ were obtained from Sinopharm Chemical Reagent (China). Horse Reddish Peroxidase (HRP) and glucose oxidase (GOx) were purchased from Beijing Dingguo Biotechnology Ltd. China. All other chemicals were analytical reagent grade or better. Solutions were prepared with deionized water (18.2 M $\Omega$, Pall Cascada).

\subsection{Apparatus}

Transmission electron microscopy (TEM) analyses were performed on a JEM-1230 electron microscope (Japan) operating at $100 \mathrm{kV}$. Absorption (extinction) spectra were measured on a Thermo Scientific NanoDrop 2000/2000 C spectrophotometer. ESI-MS were determined on a LCQ Fleet ion trap mass spectrometer (Thermo Fisher Scientific, San Jose, USA).

\subsection{Preparation of gold nanorods}

The used GNRs were synthesized using a modified method by changing the amount of $\mathrm{AgNO}_{3}$ (see the detail in Supplementary information) (Sau and Murphy, 2004).

\subsection{Experimental procedures}

All the experimental details, including detection procedure, sample preparation, have been shown in Supplementary Information.

\section{Results and discussion}

\subsection{Mechanism of the GNR-etching-based glucose sensor}

Scheme 1 outlines the mechanism of the proposed GNR-etchingbased glucose sensor. The GOx-catalyzed glucose oxidation is utilized to produce $\mathrm{H}_{2} \mathrm{O}_{2}$ which oxidized $\mathrm{I}^{-}$to $\mathrm{I}_{2}$ quickly with the catalysis of $\mathrm{MoO}_{4}{ }^{2-}$. GNRs are then etched by $\mathrm{I}_{2}$ along the longitudinal direction

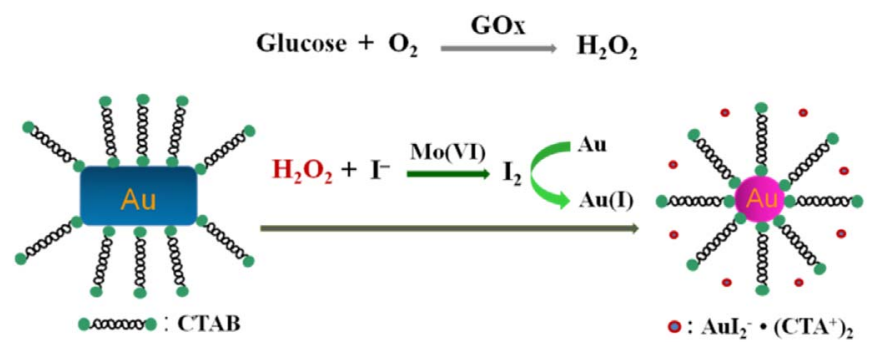

Scheme 1. Schematic representation of the mechanism of the GNR-etching-based glucose sensor. due to less surface passivation and/or the higher reaction activities at the tips of GNRs (Gill et al., 2008). The enzymatic-like etching of GNRs result in the shortening of GNRs with increase in glucose concentration (TEM images in Fig. 1). The shortening of GNRs leads to a blue-shift of the LSPR of GNRs, accompanied by a color change from blue to red (absorption spectra and photos in Fig. 1). Therefore, we can detect glucose by the spectrophotometer using the blue-shift of the LSPR absorption or by the naked eyes. Notably, the use of peak-shift for quantification can decrease the background interference.

Although high concentration of $\mathrm{H}_{2} \mathrm{O}_{2}$ can oxidize the CTABstablized GNRs, (Kou et al., 2007; Zhu et al., 2015) the low etching rate limits its analytical application. As shown in Fig. S1 (Supplementary Information), $10 \mathrm{mM} \mathrm{H}_{2} \mathrm{O}_{2}$ induced negligible change of the LSPR absorption of GNRs. Fortunately, we found iodide and $\mathrm{MoO}_{4}{ }^{2-}$ could accelerate the etching rate, (Zhang et al., 2015a) so the two reagents were introduced in this work. Fig. S2 in supplementary information shows the catalytic effects of iodide and $\mathrm{MoO}_{4}{ }^{2-}$ on the rate of etching GNRs by $\mathrm{H}_{2} \mathrm{O}_{2}$. Only addition of $\mathrm{I}^{-}$caused little LSPR absorption change and the solution kept blue in color (curve a, photo a). In contrast, the mixture of $\mathrm{I}^{-}$and $10 \mu \mathrm{M} \mathrm{H}_{2} \mathrm{O}_{2}$ induced a blue shift $(60 \mathrm{~nm})$ of the longitudinal LSPR of GNRs along with a color change from blue to grey (curve b, photo $b$ ). With further addition of $10 \mu \mathrm{M}$ $\mathrm{MoO}_{4}{ }^{2-}$, a much larger blue-shift $(100 \mathrm{~nm})$ of the longitudinal LSPR of GNRs was obtained (curve $d$ ). Simultaneously, the solution changed into red (photo $d$ ). We ascribed this to the $\mathrm{MoO}_{4}{ }^{2-}$-catalyzed oxidation of $\mathrm{I}^{-}$to $\mathrm{I}_{2}$ which further etched GNRs quickly (Copper and Koubek, 1999; Kalayci et al., 2005). As a contrast, the mixture of $\mathrm{MoO}_{4}{ }^{2-}$ and $\mathrm{I}^{-}$ caused no change of GNRs in both absorption spectrum and color (curve c, photo $c$ ). We investigated the catalytic effect of HRP on the etching of GNRs. As shown in Fig. S3 (Supplementary Information), HRP exhibited the same performance with $\mathrm{MoO}_{4}{ }^{2-}$ for accelerating the etching of GNRs. Considering the cost and the stability of reagent, $\mathrm{MoO}_{4}{ }^{2-}$ was chosen as the catalyst in this work.

To study the etching mechanism, the peroxidase-like activity of $\mathrm{MoO}_{4}{ }^{2-}$ and the etching products were carefully explored. As shown in Fig. S4 in supplementary information, $\mathrm{I}^{-}$and $\mathrm{MoO}_{4}{ }^{2-}$ or their mixture exhibited weak absorption in the absorption spectra (curves a and c). The addition of $\mathrm{H}_{2} \mathrm{O}_{2}$ to $\mathrm{I}^{-}$induced a weak peaks at 290 and $360 \mathrm{~nm}$ which are assigned to $\mathrm{I}_{3}{ }^{-}$(curve b) (Hicks and Gebicki, 1979). The further addition of $\mathrm{MoO}_{4}{ }^{2-}$ resulted in a obvious increase of the two peaks (curve d), indicating large amounts of $\mathrm{I}_{3}{ }^{-}$was produced. The formation of iodine was further confirmed by the blue color change after adding starch to the solution (Fig. S5, Supplementary Information). Similar results were obtained when the $\mathrm{MoO}_{4}{ }^{2-}$ was substituted by HRP (Fig. S6-S7, Supplementary Information). To characterize the etching product of GNRs, mass spectrum was measured. The mass spectrum (Fig. S8, Supplementary Information) indicates GNRs were transformed into $\mathrm{AuI}_{2}^{-}-\left(\mathrm{CTA}^{+}\right)_{2}$.

\subsection{Optimization of experimental conditions}

To simplify the detection procedure, we apply a "one pot" method in this work. This means the glucose-GOx enzymatic reaction and GNRs etching reaction are incubated together. Thus, the $\mathrm{pH}$ of buffer solution have to meet both glucose-GOx enzymatic reaction and $\mathrm{MoO}_{4}{ }^{2-}$ catalyzed etching of GNRs. After optimization, the $\mathrm{pH}$ of 3.8 was selected (Fig. S9, Supplementary Information). On this basis, other experimental conditions for detection of $\mathrm{H}_{2} \mathrm{O}_{2}$ were optimized afterward (CTAB $0.2 \mathrm{mM}$; KI $0.2 \mathrm{mM} ; \mathrm{MO}_{4}{ }^{2-} 10 \mu \mathrm{M}$; incubation temperature $45{ }^{\circ} \mathrm{C}$, incubation time $25 \mathrm{~min}$ ).

\subsection{Sensitivity to $\mathrm{H}_{2} \mathrm{O}_{2}$ and glucose}

Fig. S10 in supplementary information shows the absorption spectra and colors of GNRs after incubation with different concentrations of $\mathrm{H}_{2} \mathrm{O}_{2}$. The peak shift of longitudinal LSPR exhibits two linear 

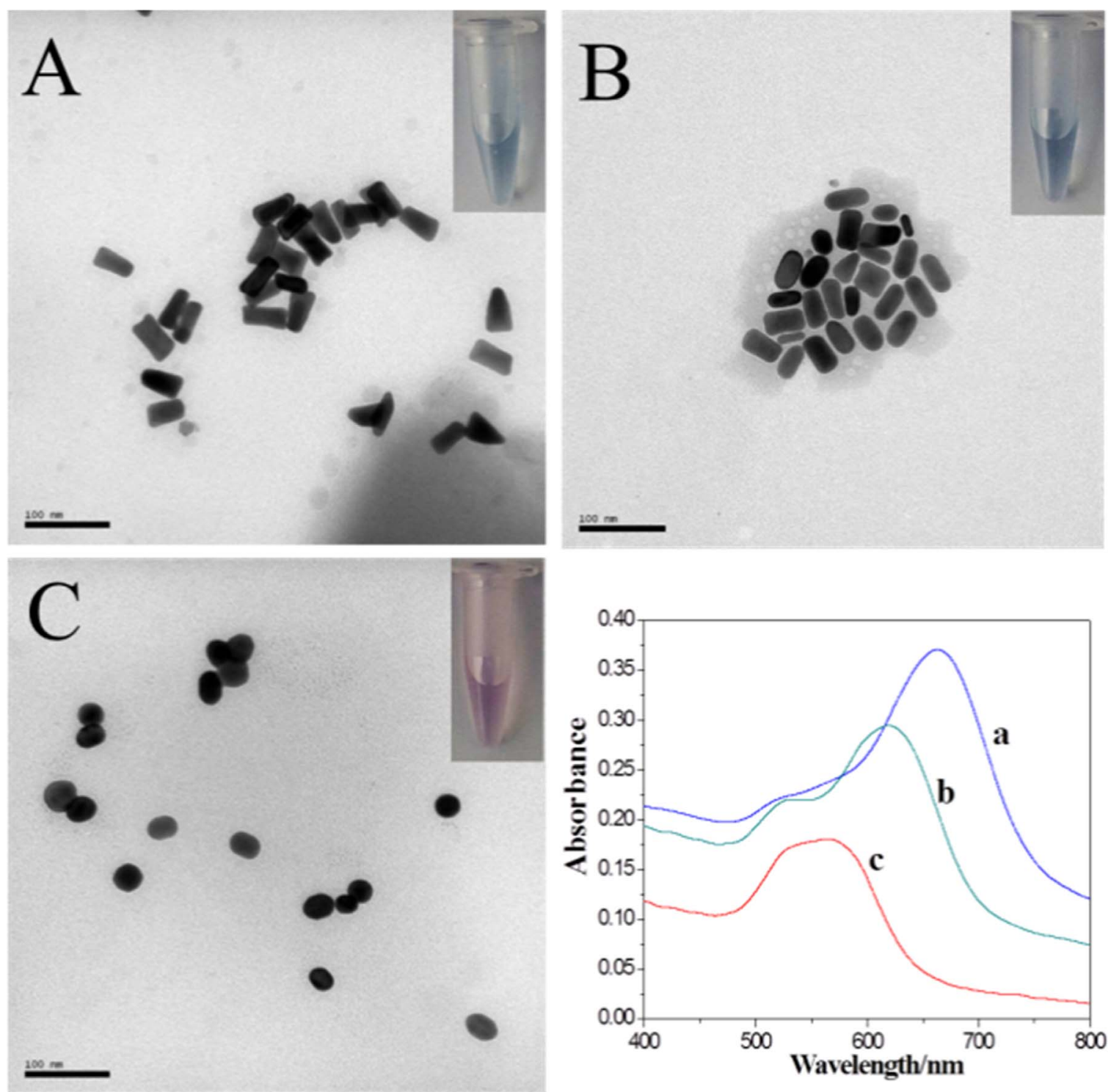

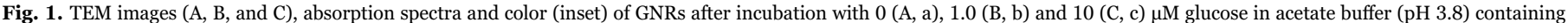
$0.2 \mathrm{mM}$ of I, $10 \mu \mathrm{M}$ of $\mathrm{MoO}_{4}{ }^{2-}$ and $0.2 \mathrm{mg} / \mathrm{mL}$ of glucose oxidase at $45^{\circ} \mathrm{C}$ for $25 \mathrm{~min}$.

relationships with the concentrations of $\mathrm{H}_{2} \mathrm{O}_{2}$ in range from 0.1 to $10 \mu \mathrm{M}$ (Fig. S11, Supplementary Information). The detection limit was calculated to be $50 \mathrm{nM}$ according to $3 \sigma$ rule. The detection limit is much lower than other plasmonic methods (Liu et al., 2014; Yang and Tseng, 2016). The excellent sensitivity to $\mathrm{H}_{2} \mathrm{O}_{2}$ will provide a good prerequisite for the ultrasensitive $\mathrm{H}_{2} \mathrm{O}_{2}$-mediated glucose detection.

Under the optimal conditions, we have carefully investigated the performance of glucose detection. As shown in Fig. 2A and B, the longitudinal LSPR absorption of GNRs shifted gradually to short wavelength with increasing in glucose concentrations. Two different linear relationships between the peak-shift and glucose concentrations were obtained, respectively. One is from 0.3 to $1.0 \mu \mathrm{M}$ (Fig. 2C) and the other is from 1.0 to $10 \mu \mathrm{M}$ (Fig. 2D). The detection limit was calculated to be $0.1 \mu \mathrm{M}$ ( $3 \sigma$ rule), which is two orders of magnitude lower than other GNRs-based glucose sensors (Liu et al., 2013; Saa et al., 2014) and is almost the lowest among nanoparticle-based glucose sensors (Table S1, Supplementary Information) (Bi et al., 2015; Bo et al., 2013; Jiang et al., 2010; Kong et al., 2014; Luo et al., 2015; Radhakumary and Sreenivasan, 2011; Shen and Xia, 2014; Shiang et al., 2009; Su et al., 2012, 2015b; Xia et al., 2013; Yi et al., 2013; Zayats et al., 2005; Zhao et al., 2015; Zheng et al., 2015). In addition, the small error bar of the data indicates the method has very high stability (Fig. 2C and D).
The digital photo ((Fig. 2E) shows the color change of GNRs with the increase of glucose concentrations from 0 to $30 \mu \mathrm{M}$. The color change as induced by $3.0 \mu \mathrm{M}$ glucose can be easily observed by naked eyes. This visual detection limit, to the best of our knowledge, is the lowest among the colorimetric methods for detection of glucose. The excellent visual sensing performance allows the on-site detection of glucose with naked eyes.

\subsection{On-site detection of urine glucose with naked eye}

When glucose concentration in urine is higher than $2.8 \mathrm{mM}$, the detection result is positive to diabetes (Urakami et al., 2008). So, it is meaningful to get a gradual color responses to "mM levels" of glucose. However, the visual detection range of the proposed method is from 3 to $30 \mu \mathrm{M}$ (Fig. 2E). After calculation, a 500-fold dilution of original sample is required (namely, $2 \mu \mathrm{L}$ of original sample is added into $1 \mathrm{~mL}$ buffer solution ( $\mathrm{pH}$ 3.8)). To get a gradual color responses to urine concentrations, different glucose concentration were spiked into a healthy human urine(its concentration was estimated to $0.45 \mathrm{mM}$ using the calibration curve in Fig. 2C). Fig. 3A shows the color responses of GNRs to different concentrations of glucose in spiked human urine. As expected, a multicolor change from blue to red to 

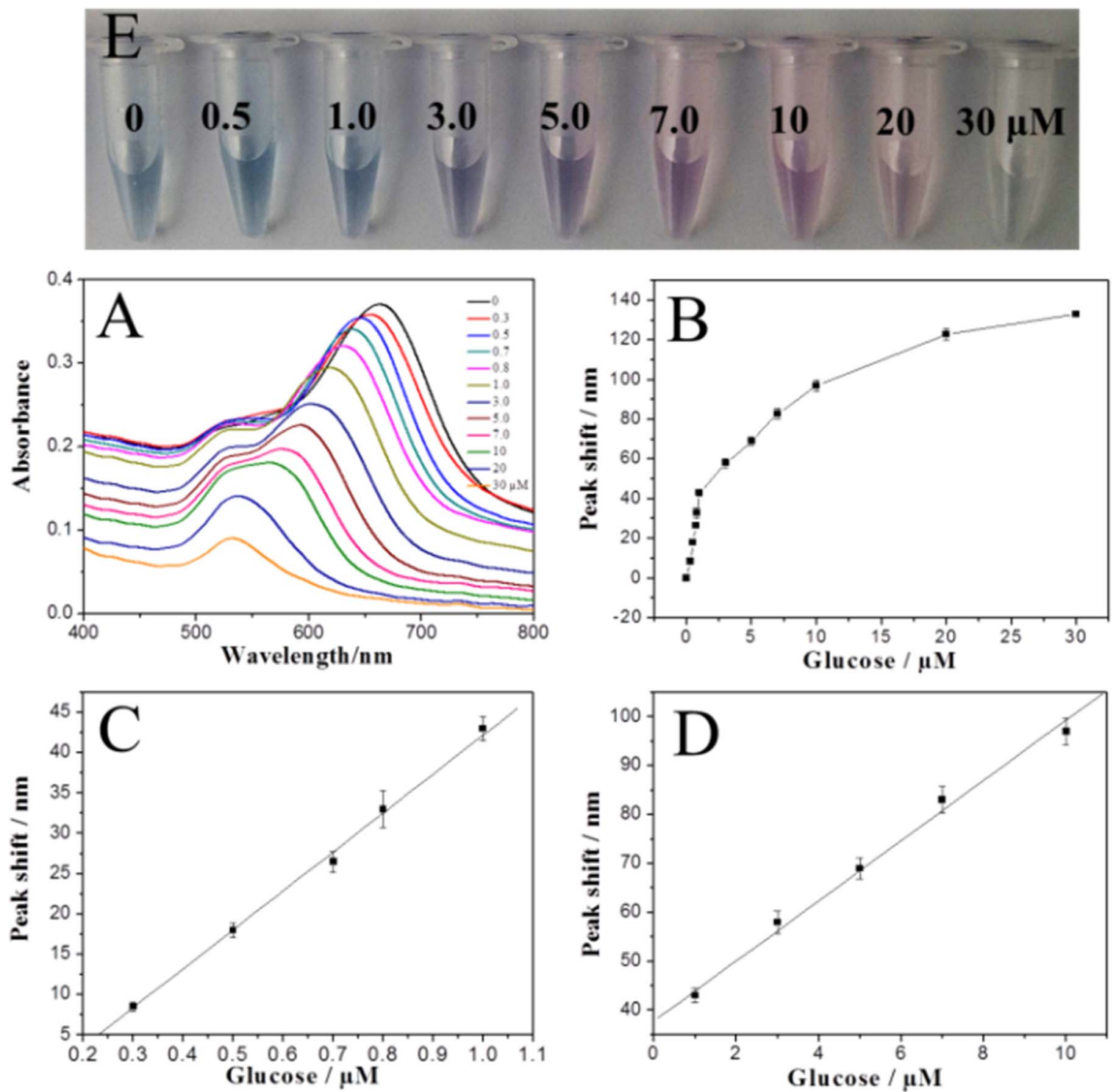

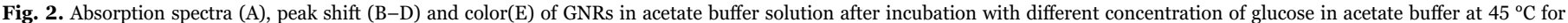
$25 \mathrm{~min}$.

A

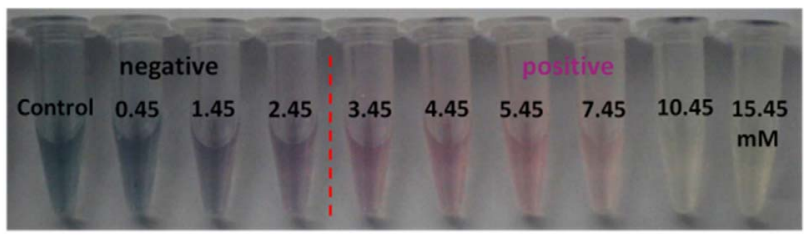

\section{$\mathrm{B}$}

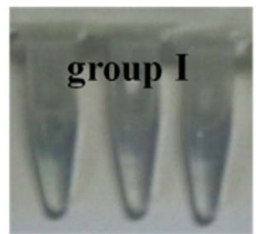

Fig. 3. Color responses of GNRs to glucose in human urine. (A) Different concentration of glucose were spiked in to healthy human urine and (B) comparison of the urine test results between 3 healthy persons (group I) and 3 diabetes patients (group II).

colorless was obtained due to the peak shift of longitudinal LSPR bands (Fig. S12, Supplementary Information). To further validate the applicability of the proposed method, urine samples from three healthy volunteers and three diabetes volunteers were tested (Fig. 3B).
Compared the results with the standard color response in Fig. 3A, we can easily distinguish healthy person and diabetes patient. All the results indicate that this method possibly provides a way for identification of the healthy status of a diabetes patient.

\subsection{Detection of glucose in human serum samples with spectrometer}

The proposed method is also used for glucose detection in human serum with appropriate pretreatment of sample (without pretreatments of serum, the proposed method is not applicable). Luckily, after removing proteins by centrifugation, satisfactory results can be also obtained with a recovery from $94.4 \%$ to $103.7 \%$ (Table S2, Supplementary Information). So, this method could provide a complementary method for accurate analysis of blood glucose.

\section{Conclusion}

We have developed a new colorimetric method for ultrasensitive detection of urine glucose based on etching of GNRs. This method is highlighted by its excellent sensitivity toward glucose with a visual detection limit of $3 \mu \mathrm{M}$, which makes it possible for the visual detection of glucose at $\mu \mathrm{M}$ levels. The successful visual detection of urine glucose 
in original sample show its applicability for on-site identification of the healthy status of a diabetes patient. Finally, the GNRs-based method for sensitive detection of $\mathrm{H}_{2} \mathrm{O}_{2}$ can be used for other $\mathrm{H}_{2} \mathrm{O}_{2}$-based sensing applications, (Chang and Ho, 2015; Liu et al., 2014; Wang et al., 2012; Yang and Tseng 2016) such as GOx-based immunoassay as shown in Scheme S1 (Supplementary Information).

\section{Acknowledgment}

We acknowledge that funding for this work from the Strategic Priority Research Program of the Chinese Academy of Sciences (XDA11020702), the Department of Science and Technology of Shandong Province (BS2009DX006), NSFC (Nos. 21275158, 21175084 and 21275091), CAS (KZCX2-YW-JS208) and the 100 Talents Program of the CAS.

\section{Appendix A. Supplementary material}

Supplementary data associated with this article can be found in the online version at doi:10.1016/j.bios.2016.09.090.

\section{References}

Bi, X., Du, X., Jiang, J., Huang, X., 2015. Anal. Chem. 87 (3), 2016-2021. Bo, H., Wang, C., Gao, Q., Qi, H., Zhang, C., 2013. Talanta 108, 131-135. Chang, H.-C., Ho, J.-aA., 2015. Anal. Chem. 87 (20), 10362-10367. Copper, C.L., Koubek, E., 1999. Inorg. Chim. Acta 288 (2), 229-232. Gill, R., Bahshi, L., Freeman, R., Willner, I., 2008. Angew. Chem. 120 (9), 1700-1703. Guariguata, L., Whiting, D., Hambleton, I., Beagley, J., Linnenkamp, U., Shaw, J., 2014. Diabetes Res. Clin. Pract. 103 (2), 137-149.

Hicks, M., Gebicki, J.M., 1979. Anal. Biochem. 99 (2), 249-253.

Jiang, Y., Zhao, H., Lin, Y., Zhu, N., Ma, Y., Mao, L., 2010. Angew. Chem. 122 (28), 4910-4914.

Kalayci, S., Somer, G., Ekmekci, G., 2005. Talanta 65 (1), 87-91.
Kong, L., Ren, Z., Du, S., Wu, J., Fu, H., 2014. Chem. Commun. 50 (38), 4921-4923. Kou, X., Ni, W., Tsung, C.K., Chan, K., Lin, H.Q., Stucky, G.D., Wang, J., 2007. Small 3 (12), 2103-2113.

Liu, D., Yang, J., Wang, H.-F., Wang, Z., Huang, X., Wang, Z., Niu, G., Hight Walker, A., Chen, X., 2014. Anal. Chem. 86 (12), 5800-5806.

Liu, X., Zhang, S., Tan, P., Zhou, J., Huang, Y., Nie, Z., Yao, S., 2013. Chem. Commun. 49 (18), 1856-1858.

Luo, F., Lin, Y., Zheng, L., Lin, X., Chi, Y., 2015. ACS Appl. Mater. Interfaces 7 (21), $11322-11329$.

Pérez-Juste, J., Pastoriza-Santos, I., Liz-Marzán, L.M., Mulvaney, P., 2005. Coord. Chem. Rev. 249 (17), 1870-1901.

Radhakumary, C., Sreenivasan, K., 2011. Anal. Chem. 83 (7), 2829-2833.

Saa, L., Coronado-Puchau, M., Pavlov, V., Liz-Marzán, L.M., 2014. Nanoscale 6 (13), 7405-7409.

Saha, K., Agasti, S.S., Kim, C., Li, X., Rotello, V.M., 2012. Chem. Rev. 112 (5), 2739-2779.

Sau, T.K., Murphy, C.J., 2004. Langmuir 20 (15), 6414-6420.

Shen, P., Xia, Y., 2014. Anal. Chem. 86 (11), 5323-5329.

Shiang, Y.-C., Huang, C.-C., Chang, H.-T., 2009. Chem. Commun. 23, 3437-3439.

Su, L., Feng, J., Zhou, X., Ren, C., Li, H., Chen, X., 2012. Anal. Chem. 84 (13) 5753-5758.

Su, L., Qin, W., Zhang, H., Rahman, Z.U., Ren, C., Ma, S., Chen, X., 2015a. Biosens. Bioelectron. 63, 384-391.

Su, L., Qin, W., Zhang, H., Rahman, Z.U., Ren, C., Ma, S., Chen, X., 2015b. Biosens. Bioelectron. 63, 384-391.

Urakami, T., Suzuki, J., Yoshida, A., Saito, H., Mugishima, H., 2008. Diabetes Res. Clin. Pract. 80 (3), 473-476.

Wang, C.-I., Chen, W.-T., Chang, H.-T., 2012. Anal. Chem. 84 (22), 9706-9712.

Xia, Y., Ye, J., Tan, K., Wang, J., Yang, G., 2013. Anal. Chem. 85 (13), 6241-6247.

Yang, Y.-C., Tseng, W.-L., 2016. Anal. Chem. 88 (10), 5355-5362.

Yi, Y., Deng, J., Zhang, Y., Li, H., Yao, S., 2013. Chem. Commun. 49 (6), 612-614.

Zayats, M., Baron, R., Popov, I., Willner, I., 2005. Nano Lett. 5 (1), 21-25.

Zhang, Z., Chen, Z., Chen, L., 2015a. Langmuir 31 (33), 9253-9259.

Zhang, Z., Chen, Z., Pan, D., Chen, L., 2014a. Langmuir 31 (1), 643-650.

Zhang, Z., Chen, Z., Qu, C., Chen, L., 2014b. Langmuir 30 (12), 3625-3630.

Zhang, Z., Chen, Z., Wang, S., Cheng, F., Chen, L., 2015b. ACS Appl. Mater. Interfaces 7 (50), 27639-27645.

Zhao, M., Gao, Y., Sun, J., Gao, F., 2015. Anal. Chem. 87 (5), 2615-2622.

Zheng, X., Zhu, Q., Song, H., Zhao, X., Yi, T., Chen, H., Chen, X., 2015. ACS Appl. Mater. Interfaces 7 (6), 3480-3491.

Zhu, Q., Wu, J., Zhao, J., Ni, W., 2015. Langmuir 31 (14), 4072-4077. 